\title{
Roots of Two Transcendental Equations as Functions of a Continuous Real Parameter
}

\author{
By Robert L. Pexton and Arno D. Steiger
}

\begin{abstract}
The roots, $\lambda$ and $\eta$, of the transcendental equations $j_{l}(\alpha \lambda) y_{l}(\lambda)=j_{l}(\lambda) y_{l}(\alpha \lambda)$ and

$$
\left[x j_{l}(x)\right]_{x=\alpha \eta}^{\prime}\left[x y_{l}(x)\right]_{x=\eta}^{\prime}=\left[x j_{l}(x)\right]_{x=\eta}^{\prime}\left[x y_{l}(x)\right]_{x=\alpha \eta}^{\prime}
$$

where $l=1,2, \ldots$ are considered as functions of the continuous real parameter $\alpha$. The symbols $j_{l}$ and $y_{l}$ denote the spherical Bessel functions of the first and second kind. The two transcendental equations are invariant under the transformations $\lambda \rightarrow-\lambda$ and $\eta \rightarrow$ $-\eta$, respectively. Therefore, only positive roots are discussed. All the $\lambda$-roots increase monotonically as $\alpha$ increases in the open interval $(0,1)$. For each order $l$, the smallest $\eta$-root decreases monotonically as $\alpha$ increases in $(0,1)$, tending towards $\sqrt{l(l+1)}$ as $\alpha$ approaches unity. For $\alpha \in(0,1)$, all the other $\eta$-roots have a minimum value equal to $\sqrt{l(l+1)} / \alpha$.
\end{abstract}

In [1] roots of the transcendental equations,

$$
j_{l}(\alpha \lambda) y_{l}(\lambda)=j_{l}(\lambda) y_{l}(\alpha \lambda)
$$

and

$$
\left[x j_{l}(x)\right]_{x=\alpha \eta}^{\prime}\left[x y_{l}(x)\right]_{x=\eta}^{\prime}=\left[x j_{l}(x)\right]_{x=\eta}^{\prime}\left[x y_{l}(x)\right]_{x=\alpha \eta}^{\prime},
$$

where $j_{l}$ and $y_{l}$ denote spherical Bessel functions of the first and second kind, are presented. Here we discuss the dependence of the roots $\lambda_{l n}$ of Eq. (1) and $\eta_{l n}$ of Eq. (2) on the continuous real parameter $\alpha$ whose domain is the open interval $(0,1)=$ $\{\alpha: 0<\alpha<1\}$. The subscript $n=1,2, \ldots$ orders the roots such that $\lambda_{l n+1}>\lambda_{l n}$ and $\eta_{l n+1}>\eta_{l n}$. Since

$$
j_{l}\left(z e^{m \pi i}\right)=e^{m l \pi i} j_{l}(z), \quad y_{l}\left(z e^{m \pi i}\right)=(-1)^{m} e^{m l \pi i} y_{l}(z)
$$

$(l, m=0,1,2, \ldots)[2$, p. $439,10.1 .34,10.1 .35]$, it follows that Eqs. (1) and (2) are invariant under the transformations $\lambda \longrightarrow-\lambda$ and $\eta \rightarrow-\eta$, respectively.

Received September 1, 1977.

AMS (MOS) subject classifications (1970). Primary 65A05; Secondary 65H05, 78A04, $33 \mathrm{~A} 04$.

Key words and phrases. Roots of transcendental equations, spherical Bessel functions, electromagnetic cavity resonators.

Notice. "This report was prepared as an account of work sponsored by the United States Government. Neither the United States nor the United States Energy Research \& Development Administration, nor any of their employees, nor any of their contractors, subcontractors, or their employees, makes any warranty, express or implied, or assumes any legal liability or responsibility for the accuracy, completeness or usefulness of any information, apparatus, product or process disclosed, or represents that its use would not infringe privately-owned rights." 
Therefore, only positive roots need be considered. If Eq. (1) is written as $F(\alpha, \lambda)=0$,

where

$$
F(\alpha, \lambda)=j_{l}(\alpha \lambda) y_{l}(\lambda)-j_{l}(\lambda) y_{l}(\alpha \lambda)
$$

then

$$
\frac{d \lambda}{d \alpha}=-\frac{\partial F / \partial \alpha}{\partial F / \partial \lambda}
$$

where

$$
\frac{\partial F}{\partial \alpha}=\lambda\left[j_{l-1}(\alpha \lambda) y_{l}(\lambda)-j_{l}(\lambda) y_{l-1}(\alpha \lambda)\right]
$$

and

(5b) $\frac{\partial F}{\partial \lambda}=\alpha\left[j_{l-1}(\alpha \lambda) y_{l}(\lambda)-j_{l}(\lambda) y_{l-1}(\alpha \lambda)\right]-\left[j_{l-1}(\lambda) y_{l}(\alpha \lambda)-j_{l}(\alpha \lambda) y_{l-1}(\lambda)\right]$.

The expressions (5a) and (5b) for the partial derivatives $\partial F / \partial \alpha$ and $\partial F / \partial \lambda$ have been obtained by means of the formula [2, p. 439, 10.1.21]

$$
\frac{l+1}{z} f_{l}(z)+\frac{d}{d z} f_{l}(z)=f_{l-1}(z), \quad f_{l}(z)=\left\{\begin{array}{l}
j_{l}(z), \\
y_{l}(z),
\end{array}\right.
$$

and by utilizing Eqs. (3). By virtue of the relation [2, p. 439, 10.1.31]

$$
j_{l}(z) y_{l-1}(z)-j_{l-1}(z) y_{l}(z)=z^{-2}
$$

and Eqs. (3) one obtains from Eqs. (4) and (5)

$$
\frac{d \lambda}{d \alpha}=-\frac{\lambda / \alpha}{1-\alpha \tau_{l}^{2}(\alpha, \lambda)}
$$

where

$$
\tau_{l}(\alpha, \lambda)=j_{l}(\alpha \lambda) / j_{l}(\lambda)=y_{l}(\alpha \lambda) / y_{l}(\lambda)
$$

For $0<\alpha<1$ expression (5b) is finite and, if Eqs. (3) hold, expression (5a) cannot vanish. Therefore,

$$
\frac{d \lambda}{d \alpha} \neq 0 \text { for } 0<\alpha<1
$$

which means that $\lambda$ is a monotonic function of $\alpha$. This implies that if, for given values of $l$ and $n$,

$$
\lambda_{l n}\left(\alpha_{2}\right)>\lambda_{l n}\left(\alpha_{1}\right) \text { and } \alpha_{2}>\alpha_{1}
$$


where $\alpha_{1} \in(0,1)$ and $\alpha_{2} \in(0,1)$, then $\lambda_{l n}(\alpha)$ is a monotonically increasing function for $0<\alpha<1$. In particular, the roots $\lambda_{l n}$ given in [1] for $l=1(1) 15$ and $n=$ 1(1)30 satisfy condition (10).

From Eq. (5a) it follows that $\lim _{\alpha \rightarrow 1} \partial F / \partial \alpha \neq 0$, and from Eq. (5b) that $\lim _{\alpha \rightarrow 1} \partial F / \partial \lambda=0$. Therefore, Eq. (4) entails that

$$
\lim _{\alpha \rightarrow 1} \frac{d \lambda}{d \alpha}= \pm \infty
$$

Condition (10) excludes the minus sign in Eq. (11).

If Eq. (2) is written as

$$
G(\alpha, \eta)=0
$$

where

$$
\begin{gathered}
G(\alpha, \eta)=s_{l}(\alpha \eta) t_{l}(\eta)-s_{l}(\eta) t_{l}(\alpha \eta), \\
s_{l}(x)=x j_{l-1}(x)-l j_{l}(x), \quad t_{l}(x)=x y_{l-1}(x)-l y_{l}(x),
\end{gathered}
$$

then

$$
\frac{d \eta}{d \alpha}=-\frac{\partial G / \partial \alpha}{\partial G / \partial \eta}
$$

where

$$
\frac{\partial G}{\partial \alpha}=\frac{1}{\alpha}\left[l(l+1)-(\alpha \eta)^{2}\right]\left[j_{l}(\alpha \eta) t_{l}(\eta)-y_{l}(\alpha \eta) s_{l}(\eta)\right]
$$

and

$$
\begin{aligned}
\frac{\partial G}{\partial \eta}= & \frac{1}{\eta}\left[l(l+1)-(\alpha \eta)^{2}\right]\left[j_{l}(\alpha \eta) t_{l}(\eta)-y_{l}(\alpha \eta) s_{l}(\eta)\right] \\
& -\frac{1}{\eta}\left[l(l+1)-\eta^{2}\right]\left[j_{l}(\eta) t_{l}(\alpha \eta)-y_{l}(\eta) s_{l}(\alpha \eta)\right] .
\end{aligned}
$$

The expression (12b) has been derived from Eq. (2) by means of Eq. (6), and the expressions (14a) and (14b) for the partial derivatives $\partial G / \partial \alpha$ and $\partial G / \partial \eta$ have been obtained by means of the formula [2, p. 439, 10.1.22]

$$
\frac{l}{z} f_{l}(z)-\frac{d}{d z} f_{l}(z)=f_{l+1}(z), \quad f_{l}(z)=\left\{\begin{array}{l}
j_{l}(z), \\
y_{l}(z) .
\end{array}\right.
$$

By virtue of Eqs. (7) and (12) the expressions (14a) and (14b) can be rewritten as

$$
\begin{gathered}
\frac{\partial G}{\partial \alpha}=\frac{1}{\alpha^{2} \eta}\left[l(l+1)-(\alpha \eta)^{2}\right] \rho_{l}^{-1}(\alpha, \eta), \\
\frac{\partial G}{\partial \eta}=\frac{1}{\alpha \eta^{2}}\left[l(l+1)-(\alpha \eta)^{2}\right] \rho_{l}^{-1}(\alpha, \eta)-\frac{1}{\eta^{2}}\left[l(l+1)-\eta^{2}\right] \rho_{l}(\alpha, \eta),
\end{gathered}
$$




$$
\rho_{l}(\alpha, \eta)=\frac{s_{l}(\alpha \eta)}{s_{l}(\eta)}=\frac{t_{l}(\alpha \eta)}{t_{l}(\eta)} .
$$

By substituting (15a) and (15b) in Eq. (13), one obtains

$$
\frac{d \eta}{d \alpha}=-\frac{\eta / \alpha}{1-\alpha \sigma_{l}(\alpha, \eta) \rho_{l}^{2}(\alpha, \eta)},
$$

where

$$
\sigma_{l}(\alpha, \eta)=\left[l(l+1)-\eta^{2}\right] /\left[l(l+1)-(\alpha \eta)^{2}\right] .
$$

The expression (16a) for the total derivative is analogous to the expression (8a). The definitions (12c) and the relations (15c) imply that $\rho_{l}(\alpha, \eta)$ and $\rho_{l}^{-1}(\alpha, \eta)$ are both nonzero. Since $\eta \neq 0$, it follows from Eq. (13) and Eqs. (15a) and (15b) that

$$
\frac{d \eta}{d \alpha}=0, \quad 0<\alpha<1,
$$

if and only if

$$
\alpha \eta=\sqrt{l(l+1)} .
$$

From Eqs. (17) and (18) it is obvious that $\eta$ is not a monotonic function of $\alpha$, unless

$$
\lim _{\alpha \rightarrow 1} \eta=\sqrt{l(l+1)}
$$

in which case one finds by means of l'Hospital's rule that

$$
\lim _{\alpha \rightarrow 1} \frac{d \eta}{d \alpha}=-\sqrt{l(l+1)} .
$$

The roots which satisfy Eq. (19) are, therefore, monotonically decreasing functions of $\alpha$. All other roots have an extremum in the open interval $(0,1)$, in accordance with Eq. (17), and satisfy

$$
\lim _{\alpha \rightarrow 1} \frac{d \eta}{d \alpha}= \pm \infty
$$

since

$$
\lim _{\alpha \rightarrow 1} \frac{\partial G}{\partial \alpha} \neq 0, \quad \text { if } \lim _{\alpha \rightarrow 1} \eta \neq \sqrt{l(l+1)}
$$

and

$$
\lim _{\alpha \rightarrow 1} \frac{\partial G}{\partial \eta}=0 .
$$

The minima of the $\eta$-roots, together with the corresponding values of the parameter $\alpha$, are presented for $l=1(1) 15$ and $n=1(1) 30$ in the microfiche supplement. In the graphs, the solid curves represent $\eta$-roots as functions of $\alpha$ for $l=1,5,10,15$ and for $n=1,2,3,4,5,10$. The dashed curves connect the minima, $\widetilde{\eta}$, of these roots. 

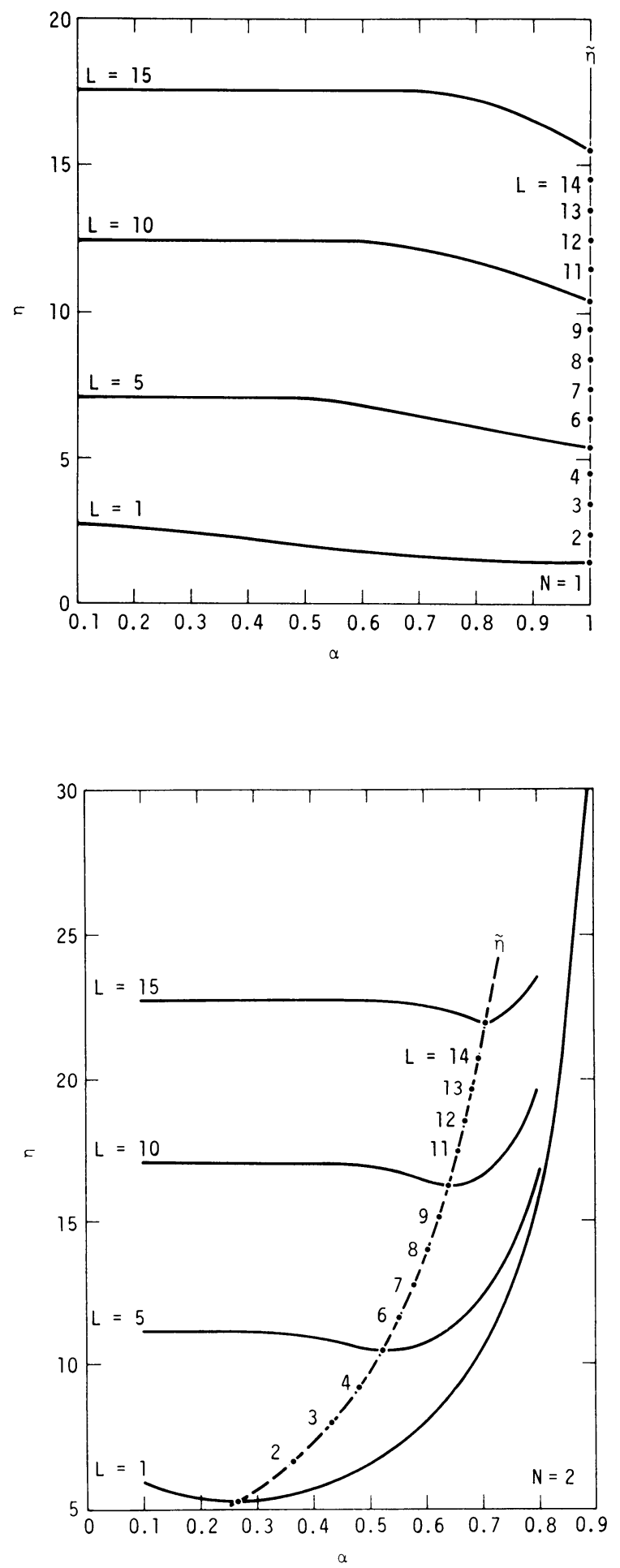

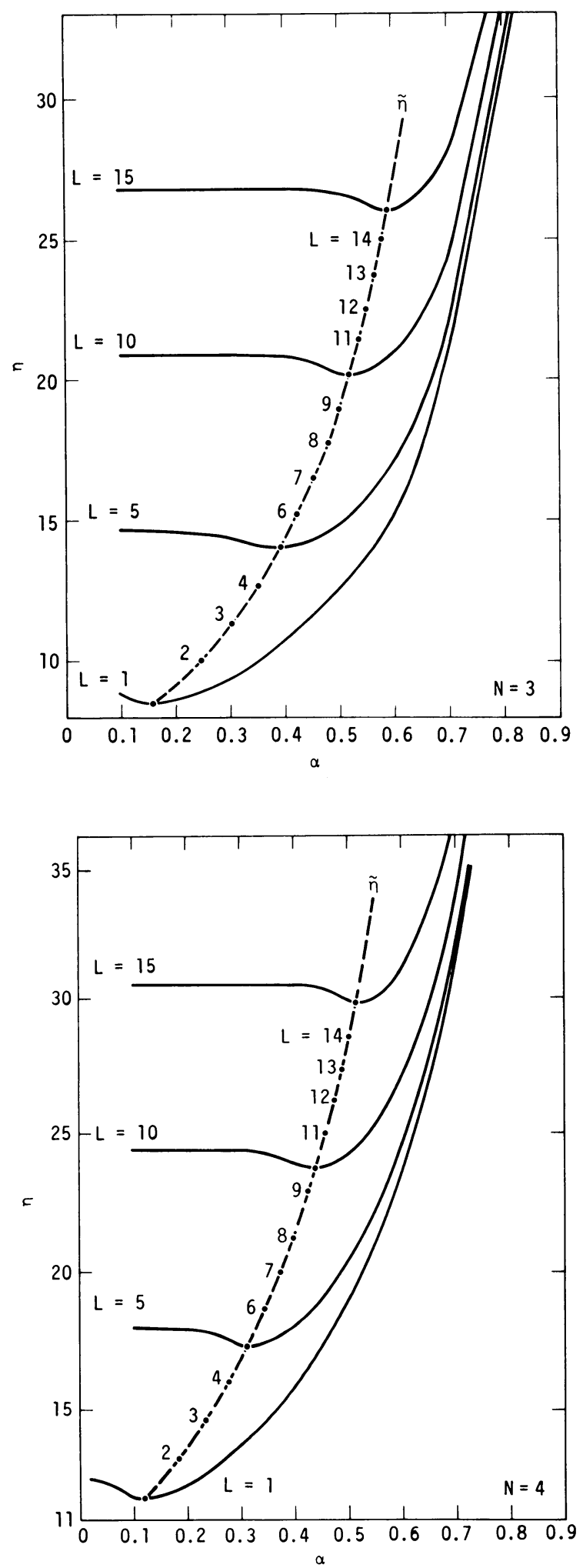

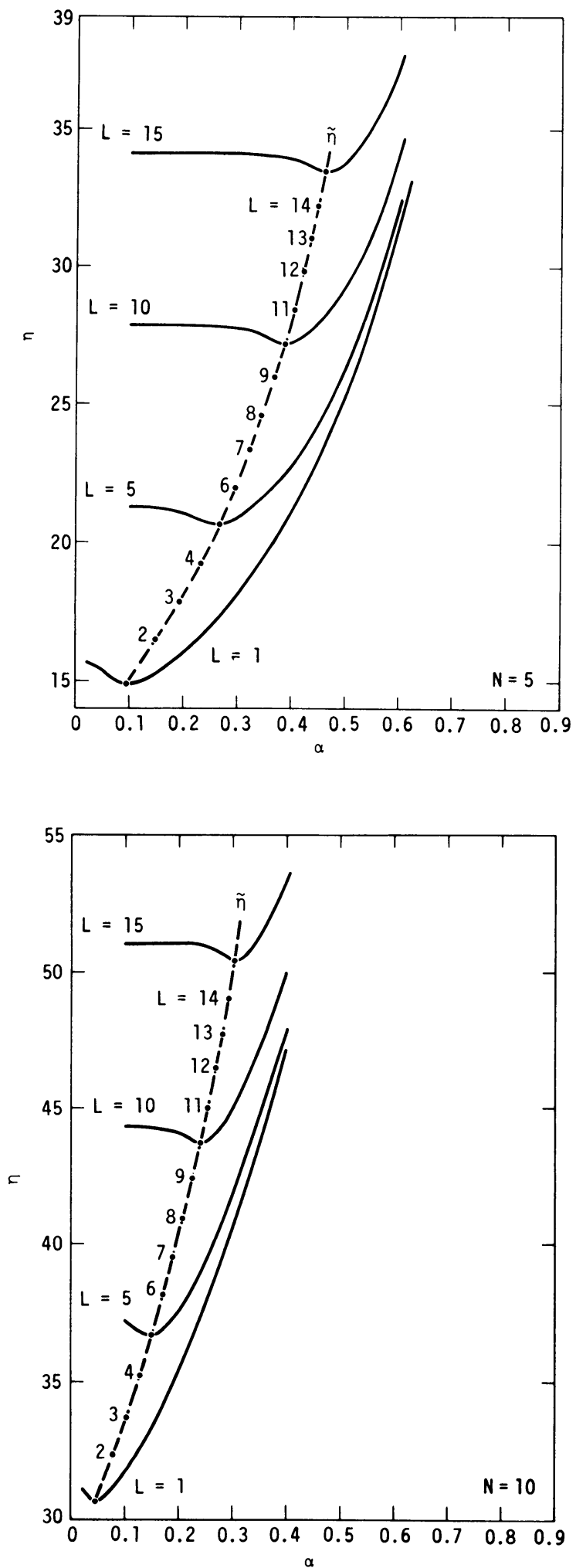
Lawrence Livermore Laboratory

University of California

Livermore, California 94550

1. ROBERT L. PEXTON \& ARNO D. STEIGER, "Roots of two transcendental equations involving spherical Bessel functions," Math. Comp., v. 31, 1977, pp. 752-753.

2. M. ABRAMOWITZ \& I. A. STEGUN, Handbook of Mathematical Functions, National Bureau of Standards Applied Mathematics Series No. 55, U. S. Government Printing Office, Washington, D. C., 1965. 